\title{
Translational and Personalized Medicine
}

\author{
KreŠIMIR PAVELIĆ, TAMARA MARTINOVIĆ \& SANDRA KRALJEVIĆ PAVELIĆ
}

\begin{abstract}
The idea behind personalized medicine is to tailor health care to an individual's unique genetic makeup. Hitherto, "one size fits all" approach was used in medicine. With the rise of personal medicine, we are moving towards a more precise, predictable and powerful medicine that is customized for each individual patient. To allow for an improvement in the acceleration and efficacy of drug development, high-throughput methods ("omics") are rapidly being developed. This leads to understanding of multiple factors that are involved in disease progress on an individual level. In order to analyze the great amount of data that is collected from such experiments, one has to turn to systems biology, an interdisciplinary science that studies complex interactions within a biological system. Finally, translational medicine comes into play, by "translating" the information gathered from research into diagnostic tools, medicines and policies, with the final goal of improving individuals' health. Personalized medicine is one of the future, and it will revolutionize the current practice of diagnosis-based medicine, once fully developed.
\end{abstract}

KEYWORDS: $\bullet$ personalized medicine $\cdot$ translational medicine $\bullet$ clinic - high-throughput methods $\bullet$ systems biology

CORRESPONDENCE AdDREss: Krešimir Pavelić, Ph.D., Professor, University of Rijeka, Department of Biotechnology, Radmile Matejčić 2, 51000 Rijeka, Croatia, email: pavelic@biotech.uniri.hr. Tamara Martinović, M.Sc., University of Rijeka, Department of Biotechnology, Radmile Matejčić 2, 51000 Rijeka, Croatia, email: tamara.martinovic@uniri.hr. Sandra Kraljević Pavelić, Ph.D., Associate Professor, University of Rijeka, Department of Biotechnology, Radmile Matejčić 2, 51000 Rijeka, Croatia, email: sandrakp@uniri.hr.

DOI 10.18690/8.25-33(2015)

ISSN 2463-7955 Print (C) 2015 LeXonomica Press

Available online at http://journals.lexonomica.press. 
K. Pavelić, T. Martinović, S. Kraljević Pavelić: Translational and Personalized Medicine

Recent achievements in life science brought out novel opportunities to monitor and asses disease progress for each individual patient. The merit for this mainly lays in the development and application of high-throughput technologies that provide global insights into genomic-proteomic profile of diseases. Biological systems are indeed complex, where biological functions are a consequence of combined activities of a multitude of molecular and cellular functions. Live systems behave non-linearly and one "input event" often causes multiple "output events". Up to now, medicine was reasonably successful in examining different diseases from a reductionist approach. However, reductionism is too limited and does not encompass fundamental questions on how do live systems function as a whole, how do they process dynamic information or how do they respond to perturbations and disease? Therefore, different approaches that might help answer these open questions are needed. New accomplishments in high-throughput technologies, e.g. transcriptomics that provide an entire insight into gene activity in an organism, proteomics that provide for knowledge on global protein profiles or metabolomics that provide information on metabolite status, will dramatically change molecular medicine and life science (Bošnjak, Pavelić and Kraljevic Pavelic 2008). Here, we must have in mind that genes and proteins cannot explain everything. One needs to consider other complex elements including molecular pathways, protein structure, secondary protein modifications, epigenetics and many others. New methods to provide some novel insights into biological mechanisms may be lipidomics, glycomics, metabolomics, nutrinomics and even complex structural genomics methodologies and approaches. The use of these methods in medicine may allow an individual approach towards each patient and boost the turning point in medicine from hitherto approach that is based on new drug discovery, to a future, more preventive approach (Simmons et al. 2012). This approach will bring substantial social shifts that will change socio-humanistic relationships and raise a whole series of important questions: moral-ethical, legal and socio-economic (Feldman 2011; ESF Forward Look 2012). This will result from current challenges in medicine and humanity that are both faced with multiple processes of globalization and fast changes in society. Some of the current issues are indeed, new severe and fast spreading infectious diseases, certain diseases change their "behavior pattern", aging of the population brings demographic change and fast and dramatic climate changes (ESF 2012; McCarty et al. 2005).

When we use the term personalized medicine, it implies the systematic use of information about the individual patient, with the goal to choose optimal prevention and/or welfare therapy. Introduction of high-throughput methods into medicine allows for the creation of true personalized medicine (in other words precise medicine) because it ensures the application of genomic and proteomic research, directly or indirectly, for prevention and individually-tailored treatment (Mirnezami, Nicholson and Darzi 2012). Therefore, major focus of personalized 
medicine in current medical treatments is to generate innovative treatments and drugs, as well as to reduce the negative effects of the drugs (ESF Forward Look 2012; McCarty et al. 2005; Ng et al. 2009).

\section{High-throughput analytical methods and personalized medicine}

Progress in high-throughput analytical methods will be extremely important for further development of personalized medicine. Although global methods for biological analyses are still in their infancy, their role in acceleration and improvement of the drug development process is highly probable. Global analyses are indeed extremely powerful when deep elucidations of biochemical pathways are required, as they provide effective means to explain diseases pathogenesis mechanisms and drug distribution patterns in the body. Considering that expenses for drug development are constantly increasing, while the number of newly approved drugs is decreasing and many patients (e.g. cancer patients) cannot be treated efficiently and securely, global analyses might efficiently provide novel solutions to these issues (Hamburg and Collins, 2010).

Application of high-throughput analytical methods in biomedicine therefore allows for comprehension of factors involved in disease development on the individual level, for each patient. This kind of personalization imposes a great turning point in medicine where recognition of factors that precede the individual's illness is finally possible (Bošnjak, Pavelić, Kraljevic Pavelic 2008; Spira et al. 2007). The drift from global to personalized medicine will radically change the health system and will generate new and innovative ways of treatment, reduce negative side effects of therapy, increase the quality and reduce expenses of clinical care, and finally, allow for a better doctor-patient relationship. Global genome and proteome analyses are growing increasingly and are improving both from the technological side and methodologically. Their use in clinical medicine has already started. One can freely state that the upcoming era of high-throughput methods allows for a truly personalized approach to the patient and a change of the medical paradigm - from medicine, founded mostly on treatment, to preventive medicine or pre-symptomatic medicine.

It is expected that high-throughput methods and nanomedicine, which represents the technological basis of "omics", will give a noticeably more personal approach to treatment of many diseases, an increase in the efficiency of pharmaceutical therapy, and a reduction of adverse drug effects.

"Omics" methods - transcriptomics, proteomics, metabolomics, lipidomics, glycomics, structural genomics etc., are based on nanotechnologies. "Omics" are considered as global methods for characterization of all or a majority of members belonging to a certain family of molecules, within one experimental step or analysis. Transcriptomics represent a systematic analysis of all genes in an 
organism while proteomics refers to a systematic analysis of protein expression under specific conditions which include separation, identification and characterization of proteins in an organism. The term proteome originates from the definition 'Protein complement to a genome', postulated in 1994, and refers to all proteins that a genome expresses throughout the life (Sadée 2005). The proteome importance might be explained in a biological context where functional parts such as molecular complexes, signaling networks and whole organelles are important regulators of cell processes. Proteins are individual components of these functional parts and have multiple levels of regulations which include protein "circulation" (recycling and degradation), post-translational modifications, subcellular localization and protein-protein interactions. The latter lead to a formation of complexes that are crucial in cell signaling or cell architecture. In order to obtain biologically relevant insights into the disease's molecular nature and develop a so called "cell map", it is important to integrate the knowledge obtained through comprehensive high-throughput studies, especially genomics and proteomics. The potential benefit from understanding processes based on the proteome is unquestionable because it offers possibilities of diagnosis, classification, prognosis and estimation of the therapeutic effect, and finally leads to a true personalized medicine based on patient's proteomic profile (WhirlCarrillo et al.2012; Longo 2012).

Just as within clinical research, studies required to provide robust protocols for personalized medicine include analysis of data collected from large population studies (groups of persons who share the same or similar characteristics, e.g. place of birth and living area, their age, etc.) and collecting of biological samples ('biobanks'). The combination of carefully classified biological samples and detailed relevant clinical information collected from 'bio-banks' are key components of modern research infrastructure for classification of subtypes of ailments, and will stimulate development of personalized medicine in the $21^{\text {st }}$ century.

Data from hundreds and thousands of people should be analyzed in order to elucidate factors that provide for individual differences. These factors include environmental and life-style factors as well. These studies are extremely expensive and the results should be handled correctly and professionally. Results of such studies are vital for development of personalized medicine. Gathering of data on phenotype, diagnostic criteria, life-style and environment, all factors that are changing throughout life, should be improved. Furthermore, with the advance of knowledge of human biology new questions arise, and demand on new studies involving new test subject groups and demographic data, which may not exist in the previously conducted experiments, is raising. It seems, therefore, that renewal and improvement of phenotypical and environmental databases are urgently needed in order to improve research oriented towards true personalized medicine. 


\section{Translational medicine, systems biology and personalized medicine}

Systems biology is a new interdisciplinary scientific field that encompasses biology, mathematics, computer science, physics, engineering and other disciplines. Majority of biological systems are too complex to model or visualize even for most powerful computer simulations that cannot provide for all attributes at once. A usable model should be, thus, able to provide reliable predictive values and we believe that a certain degree of abstraction when approaching such problem, which is directed to systemic behavior of importance, is an imperative. Within this field, global experimental approaches have already been integrated into biology and clinical practice. Such approach changed previous clinical descriptions of many diseases and new molecular signatures are implemented in identification of new disease subtypes and prognostic standards, e.g. in oncology. This approach is used both for characterization of disease type and prognosis, and for prediction of clinical response. In that way, a physician has additional options to assign and choose appropriate therapy/chemotherapy and decide whether to use 'target agents' in treatment of cancer patients (Longo 2012; Parkinson and Johnson, 2012). Targeted cancer therapy faces gross challenges at the moment. Since the marketing of the first "smart" drugs (trastuzumab, cetoksimab, erlotinib), which was followed by an explosion of transcriptomic, proteomic, genomic and RNA-interference (RNAi) studies related to therapy outcomes, an increasing amount of data is being gathered related to drugs' mechanisms of action and biological response to therapy. Here, indispensable systematization and processing of large amounts of data is obvious. This requires constant improvement in the processes of data storage, modelling and analysis, all covered within the systems biology approach. The vast amount of data still remains somewhat interpretable.

The application of systems biology on drug discovery mainly against cancer is based on convergence of three trends: aimed therapy, wide systematic experimental platform and advance in information technology. This convergence has already a strong effect on practical clinical medicine. Applying such approach to development of next generation drugs, which should improve the treatment of each individual patient, is, however, a challenge (Yan 2011). Systems biology ensures an integrative approach to drug discovery which leads to better decision making. Some of the benefits are related to knowledge on choosing the right targets, choosing the right model to study target(s), understand the role of target(s) and disease pathways, and making better decisions in planning and realization of clinical drug usage.

Today we understand that systems biology as a discipline bears important roles in drug discovery: identification of targets by use of clinical and genomic data, identification and implementation of clinically relevant bio-markers based on preclinical studies, implementation of quantitative models as well as models that 
can be tested for hypotheses-testing connected to dosage, combining and stratification of patients. For example, cancer represents a group of mostly acquired diverse genetically predisposed diseases. Here, clinical samples, and not cell lines or system models, contribute to the discovery of targets with clinical importance. The true challenge in target identification lies in giving proof that the target is relevant and connected to cancer. Thus, integrative clinical genomic studies are a good starting point for further confirmations of the system model. Observations show that genomic deregulation patterns conserved in the tumor sample or cell line are probably not irrelevant data.

A good example of using system models is breast cancer. Molecular data are the starting point and the main driving force for a personalized medicine approach. During the last years, a vast number of clinically-genetic data on breast cancer before, during and after therapy has been published. This information was used to estimate the relevance of disease subtypes, which was not possible by sole use of classical pathology classification. Data is integrated within meta-analytical studies and new targets are identified where functional "signatures" for key tumorsuppressor pathways, such as p53, have gained diagnostic and therapeutic importance. Clinical data is used in combination with cell line models with the purpose to identify relevant subgroups of models sensitive to targeted agents. Interpolation of genomic data from both models is already implemented in some clinical laboratories where patients' samples and primary cell lines are used within the translational approach. However, tumor cell lines are not yet adequately predictable, if the clinical response is considered. Furthermore, human cell lines transfected into nude mice are also a useful in vivo model, but again bring a limited predictive value with regard to the therapeutic response. Implanting patient's tissue into nude mice might be an alternative to this model. Combined with genomic data, these models represent an attractive platform for transfer (translation) from discovery to clinic (Longo 2012; Chin, Andersen and Futreal 2011).

\section{$4 \quad$ Transfer from discovery to clinic}

Ever since the first experimental (transcriptomic) disease profiling studies, systems biology approach has improved towards therapy routing by use of genomics data. Some of the results in this field have a major impact in oncology clinical practice where disease subtypes are defined by use of limited microarray tests that drive the onset of a right clinical decision.

Genetic "signatures" are indeed useful in identification of molecular subtypes and prediction of chemo-sensitivity and treatment outcome. Integrative genetic studies were shown to be useful in predicting genetic lesions when conducted on a large number of cases. Genetic lesions are certainly more complex and widespread than generally presumed. Implications of such studies are great and will provide for the development of dynamic models related to genetic progression of cancer growth. 
Discovery and development of new drugs cannot be directly designed by results obtained from high-throughput studies. Instead, we are constantly faced with the challenge on how to interpret the data and predict the outcome of a new drug or treatment, probably acting through yet unknown mechanisms of action. Integrative systems biology can provide data on pharmacodynamic patterns and biomarker modulation. This might well be used for assessment of biologically effective dosages and identification of system models combined with genomic data in specific patients' groups or individual patients which are most prone to react to therapy.

At last, it may be concluded that system based approach to translational medicine is possible but requires intertwining of several disciplines - informatics science, post-genomic disciplines and biomedical sciences, working all together towards a mutual goal that is understanding the complexity of disease pathogenesis and patients' treatment. Molecular routing towards tumor aberrations has become a standard on which modern treatment rests. One has to keep in mind that individual molecular targets do not give a full picture of a disease, due to disease complexity, a number of deregulated molecular networks and complex interactions between altered cells and the surrounding micro-environment. The approach based on systems biology offers a more direct congregation of platforms for noticing phenomenological diversities of disease, inlcuding cancer, and leads to a much wider range of experiments as well as more relevant clinical models.

\section{Conclusion}

Personalized medicine and the whole movement towards personalized approach to the patient highly relying on high-throughput analyses, might significantly change the quality of life. How to confront potential problems is still not completely understood. This should be tackled by socio-humanistic sciences and their power. Even though our opinion is that scientists from social fields should be involved in research and system analyses within personalized medicine, we still see lack of readiness for this challenging interdisciplinary and transdisciplinary approach. More generally, modern scientists should learn much more and enter into the field of their science colleagues and likewise. Today's degree of knowledge, even though highly specialized and sophisticated, does not prepare the scientists, neither is sufficient to cope with such societal challenges. We are witnesses of deflection of science from hitherto reductionist towards an integrated outlook on life. In accordance, health care will experience radical changes with the dramatic development of biomedical research. It is to be expected that medicine of the future will be personalized and preventive, more efficient and cheaper. 


\section{References}

Bošnjak, H., Pavelić, K. \& Kraljevic Pavelic, S. (2008) Towards preventive medicine, EMBO Reports, 9(11), pp. 1056-1060, doi: 10.1038/embor.2008.198.

Chin, L., Andersen, J. N. \& Futreal, P. A. (2011) Does comparative-effectiveness research threaten personalized medicine? Nature Medicine (2011), 17: 297-303.

ESF Forward Look (2012) Personalized medicine for the European citizen. Toward more precize medicine for the diagnosis, treatment and prevention of disease (iPM), (Strasbourg: European Science Foundation), available at: http://www.esf.org/uploads/media/Personalised_Medicine.pdf (March 27, 2015).

Feldman, E. A. (2012) The Genetic Information Nondiscrimination Act (GINA): Public policy and medical practice in the age of personalized medicine, Journal of General Internal Medicine, 27(6), pp. 743-746, doi: 10.1007/s11606-012-1988-6.

Hamburg, M. A. \& Collins, F. S (2010) The path to personalized medicine, New England Journal of Medicine, 363(4), pp. 301-304, doi: 10.1056/NEJMp1006304.

Katsios, C. \& Roukos, D. H. (2010) Individual genomes and personalized medicine: life diversity and complexity, Personalized Medicine, 7(4), pp. 347-350, doi: 10.2217/pme.10.30.

Kraljević, S., Stambrook, P. \& Pavelić, K (2004) Accelerating drug discovery, EMBO Reports 2004, 5(9), pp. 837-842, doi: 10.1038/sj.embor.7400236.

Longo, S. L. (2012) Tumor heterogeneity and personalized medicine, New England Journal of Medicine, 366(10), pp. 956-957, doi: 10.1056/NEJMe1200656.

McCarty, C. A., Wilke, R. A., Giampietro, P. F., Wesbrook, S. D. \& Caldwell, M. D. (2005) Marshfield Clinic Personalized Medicine Research Project (PMRP): design, methods and recruitment for a large population-based biobank, Future Medicine, 2(1), pp. 49-79, doi: 10.1517/17410541.2.1.49.

Mirnezami, R., Nicholson, J. \& Darzi, A. (2012) Preparing for Precision Medicine, New England Journal of Medicine, 366 (6), pp. 489-491, doi: 10.1056/NEJMp1114866.

Ng, P. C., Murray, S. S., Levy, S. \& Venter, J. C. (2009) An agenda for personalized medicine, Nature, 461, pp. 724-726, doi:10.1038/461724a.

Parkinson, D. R., Johnson, B. E., Sledge, G. W. (2012) Making Personalized Cancer Medicine a Reality: Challenges and Opportunities in the Development of Biomarkers and Companion Diagnostics, Clinical Cancer Research, 18(3), pp. 619-624, doi: 10.1158/1078-0432.CCR-11-2017.

Sadée, W. \& Dai, Z. (2005) Pharmacogenetics/genomics and personalized medicine, Human Molecular Genetics, 14(2), pp. R207-R214.

Simmons, L. A., Dinan, M. A., Robinson, T. J. \& Snyderman, R (2012) Personalized medicine is more than genomic medicine: confusion over terminology impedes progress towards personalized healthcare, Future Medicine, 9(1), pp. 85-91, doi: 10.2217/pme.11.86.

Spira, A., Beane, J. E., Shah, V., Steiling, K., Gang Liu, G., Schembri, F., Gilman, S., Dumas Y.-M., Calner, P., Sebastiani, P., Sridhar, S., Beamis, J., Lamb, C., Anderson, T., Gerry, N., Keane, J., Lenburg, M. E. \& Brody, J. S. (2007) Airway epithelial gene expression in the diagnostic evaluation of smokers with suspect lung cancer, Nature Medicine, 13(3), pp. $361-366$.

Yan, Q. (2011) Toward the integration of personalized and systems medicine: challenges, opportunities and approaches, Personalized Medicine, 8(1), pp. 1-4, doi: 10.2217/pme.10.77.

Weston, A. D. \& Hood, L. (2004) Systems Biology, Proteomics, and the Future of Health Care:Toward Predictive, Preventative, and Personalized Medicine. Journal of Proteome Research, 3(2), pp. 179-196. 
Whirl-Carrillo, M., McDonagh, E. M., Hebert J. M., Gong, L., Sangkuhl, K., Thorn C. F., Altman, R. B. \& Klein, T. E. (2012) Pharmacogenomics knowledge for personalized medicine, Clinical Pharmacology \& Therapeutics, 92(4), pp. 414-417, doi: 10.1038/clpt.2012.96. 